\title{
Visualization platform design of failure outage risk assessment based on GIS
}

\author{
Peng Liu ${ }^{\dagger}$, Wan-Xing Sheng, Guang-Xian Lv, \\ Distribution Department, China Electric Power Research Institute, \\ Beijing, 100192, China \\ †E-mail: liupeng1@epri.sgcc.com.cn \\ Xian-Guo Kang, Wei Meng, \\ Handan Supply Branch, State Grid Hebei Electric Power Company, \\ Handan, 056001, China \\ Zi-Jin $\mathrm{Li}$ \\ Electric Power Research Institute, State Grid Beijing Electric Power Company, \\ Beijing, 100075, China
}

\begin{abstract}
With the development of science and technology, a large amount of intermediate data is generated by computer needed to process by visualization technology, in order to achieve computer-aided analysis, reproduction of the objective. Modern data visualization refers to translate the data into the graphics or images displayed on the screen for interactive processing the theory and method by computer graphics and image processing technology. In order to achieve the visualization on failure outage risk assessment, the visualization platform was built to satisfy the requirements of 3D GIS, virtual reality and intelligent interaction. It's aimed that the smart grid can be understood and interactive, to construct and design with visual service, intelligence perception and decision support for the three major characteristics of distribution network visualization platform.
\end{abstract}

Keywords: Distribution Network; Failure Outage; GIS; Risk Assessment; Visualization Platform.

\section{Introduction}

In the traditional application system of distribution network, there are two main types of graphics tools. One is displayed by line diagram, and the others are generally developed by themselves. The line diagram mainly shows logic relation of electrical connection. It's not care about the specific location, but the topology and the corresponding measurement value and the switch opening and closing state. The dispatchers usually get information which the operation of the power distribution network through the data displayed on graph or geographic maps. The geographic information system (GIS) is generally commercial, it 
display device geographical location and terrain. GIS is used for inspecting equipment conveniently and understanding line direction.

Research shows that the accuracy rate is $59 \%$ which memories of line diagram, but it's $85 \%$ which memories of analysis chart. It reminds us that the line diagram is not the most effective way for dispatchers. It should be introduce a new display technology. With the improvement of computer hardware operation speed and 3D software technology, it can be well displayed the 3D graphics through the basis of need not add any hardware, to achieve humancomputer interaction, such as the simulation application of 3D substation. The paper uses the GIS technology to design a visualization platform of failure outage risk assessment, provide feasible verification and can be implemented method for failure analysis and judgment of distribution network operation.

\section{Platform Design}

The main function of visualization platform includes creating scenes, creating different types of window, adding object, modifying the object style, activating the window and so on. Through the unified data interface custom released to the Web Service interface for other systems or program calls according to different application scenarios. In order to support different business application system or different applications scene, the platform should be designed as business of independent, so it designs multiple scenarios used for the basic window type, GIS object model, and visual object model.

\subsection{GIS data object design}

The complex geography objects generally abstract several geometric types, such as point, line, polygon (surface) etc. This platform customs VGS format, and defines several basic geometric elements by Geometry abstract elements. It provides a point, line, ring, polygon, 3D model and other basic geometric shapes, which can also define complex geometry by MultiGeometry basic geometric aggregation of different forms of composite geometry.

\subsubsection{Point object element model}

In VGS specification, geometry of the point object is described as:

$<$ Point id="ID_XXXX" targetId="ID_XXXX">

$<$ coordinates $>$ longitude, latitude, height $</$ coordinates $>$

$</$ Point $>$ 


\subsubsection{Line object topology model}

The VGS specification defines two lines: one is the multi segment line, and the other is a ring, namely a polyline. Their grammatical structure is the same, except that the start point and end point coordinates of the same ring, but line is not. For example, longitude 1- longitude N, latitude 1-latitude N, height 1height N. In the VGS specification, geometry of the line object is described as:

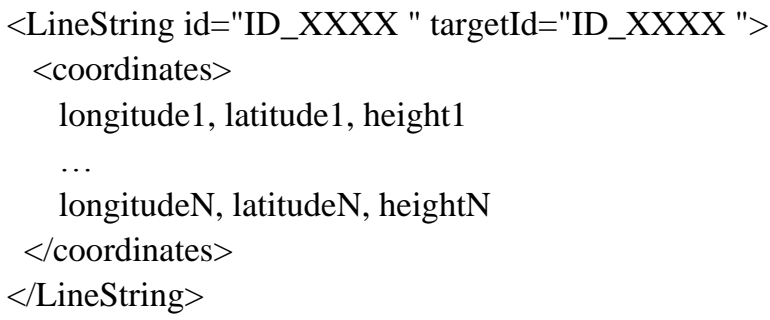

\subsubsection{Surface object area model}

The face that VGS specification expressed as the polygon domain, the outer boundary is defined by outerBoundaryIs, and the inner boundary of island area defined by innerBoundaryIs. It shows that the no area region is a special case of area region. In the VGS specification, geometry of the surface object is described as:

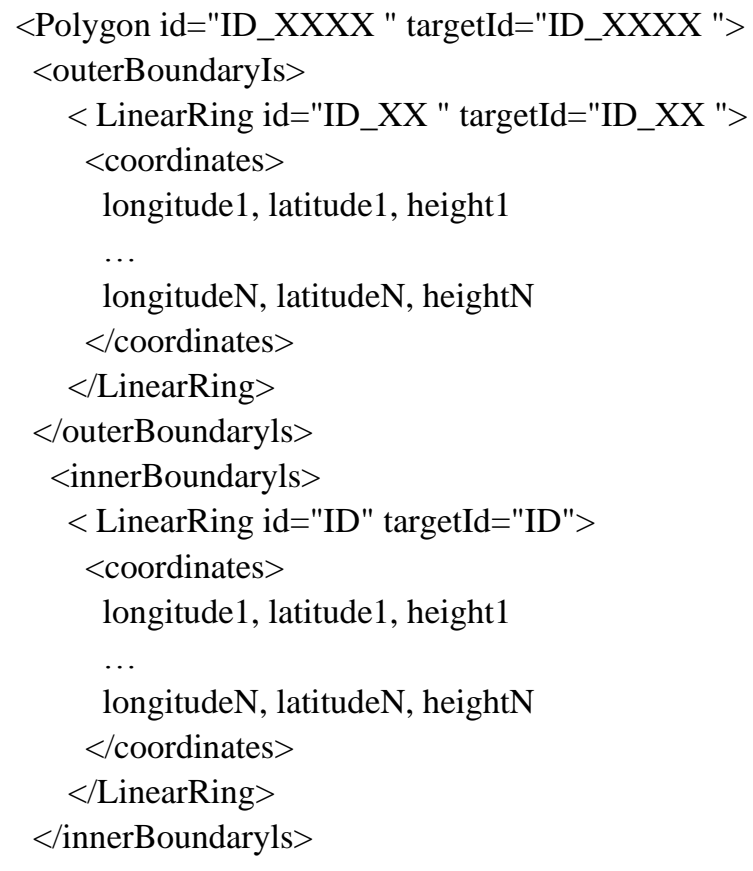


$<$ Polygon $>$

\subsection{Failure outage risk assessment application demonstration scenario design}

It generally includes that the creation of three-dimensional sphere window, three-dimensional plane window, DEM window and SVG window terrain in power failure risk assessment service application scene, then adding the point of different objects through different window and visual object, in order to achieve the power failure risk assessment to demonstrate. The scene of the main application contains:

\subsubsection{Sphere of three dimensional}

Take whole country as the goal, it shows risk information level of each city as the unit of city, and shows suggesting that the effect of different risk values of the city through the column of visual object height, color, combined with the dynamic aperture scintillation, in order to select the target interaction shows its risk numerical information.

\subsubsection{Flat surface three dimensional}

Take whole city as the goal, substation and line as the object, it shows 3D model of substation on the map, and shows the reliability of substation by numerical, alarm animation of substation by aperture. According to the substation, it draws line by different color, using bright to eye-catching, using animation to direction of current, using different aperture scintillation color to hint alarm and outage.

\subsubsection{DEM landform}

Take a single or multiple feeders as the goal, it shows data corresponding to the feeder line diagram through SVG single line diagram window, shows different colors to distinguish the line outage alarm circuit by no image map interface.

\subsection{Panoramic visualization model for distribution network design}

To carry out the power failure risk assessment in addition to the fusion of multisource data, also needs a key characteristic of the dispatcher friendly, to realize dispatcher's requirement. In order to achieve unified system design process, task definition, risk assessment, dispatcher friendly display mode and so on. 


\subsubsection{Operation data of distribution network visualization}

The advantage of single line diagram is clear and regular structure, it can be node data and line data clearly marked to the corresponding location. Because of corresponding with power wiring diagram and geographical location in actual system, it's very intuitive.

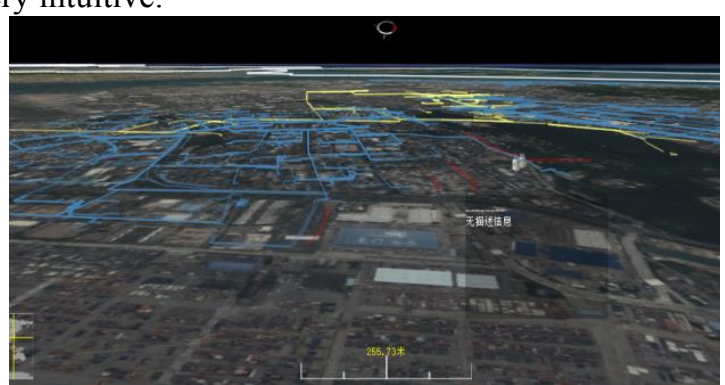

Fig. 1. Example of power operation data visualization

\subsubsection{Equipment visualization}

It's used for equipment management, environmental management, disaster prevention management, planning and configuration of each module report display in asset management, and the equipment state monitor display and so on. It also can display the failure equipment in the station and the associated line and station quickly in the geographical wiring diagram, in order to timely equipment failure processing. Especially visualization of transformer, which information can be presented in front of the staff includes hot spot temperature, transformer life and online load limit.

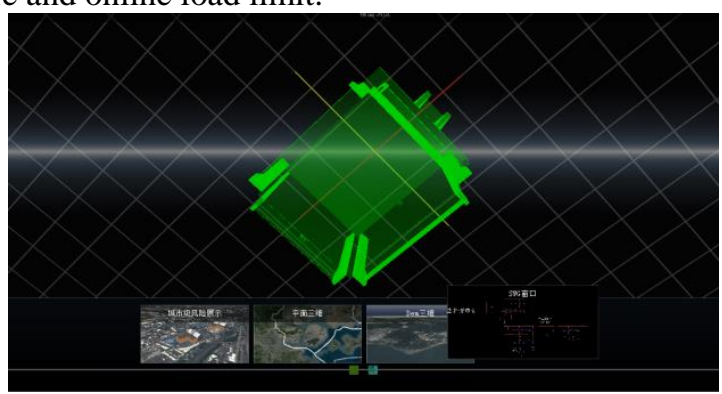

Fig. 2. Example of equipment visualization

\subsubsection{Contour diagram}

It's the same with the numerical diagram of the same color. The principle of contour display is continuously displaying data in the pixel of window, which value express an object of power system. The contour can be continuous, also can be discrete. 


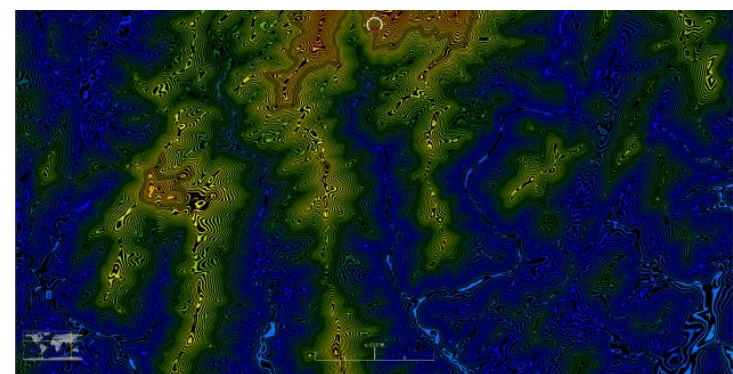

Fig. 3. Example of contour diagram

\subsubsection{Area coloring diagram}

According to the voltage of each region on overall situation to fill different colors in geographical system diagrams. It can immediately identify which region voltage is normal or not, and which regional voltage is abnormal through the color.

Fig. 4. Example of area coloring diagram.

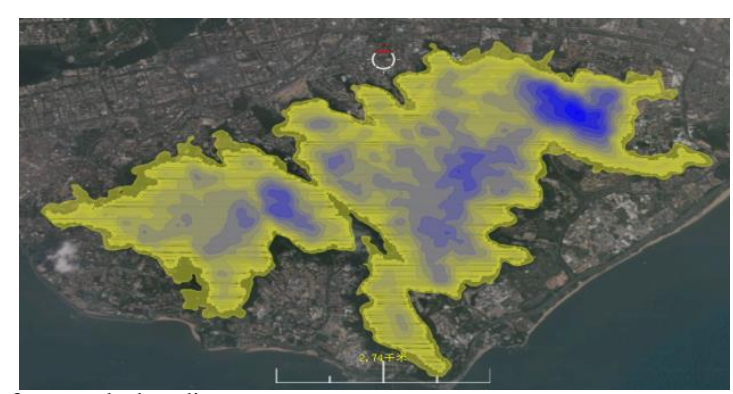

\subsubsection{Bar diagram}

It expresses system data through the two-dimensional or three-dimensional cylindrical, with the value for height, and with beyond the mark or not for color. Bar diagram is displayed in geographic diagram or line diagram, or independent displayed. Height and color of bar diagram change colors with the change of equipment information.

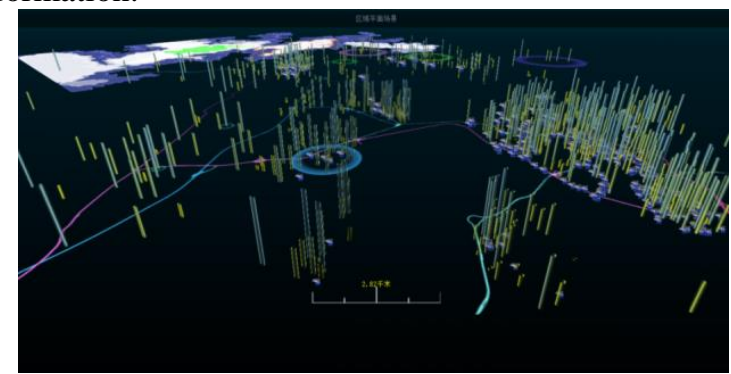

Fig. 5. Example of bar diagram 


\subsection{6. $3 D$ surface}

In the three-dimensional space, it expressed distribution change of the voltage value through the high and low in the curve surface combined with colors.

Fig. 6. Example of 3D surface

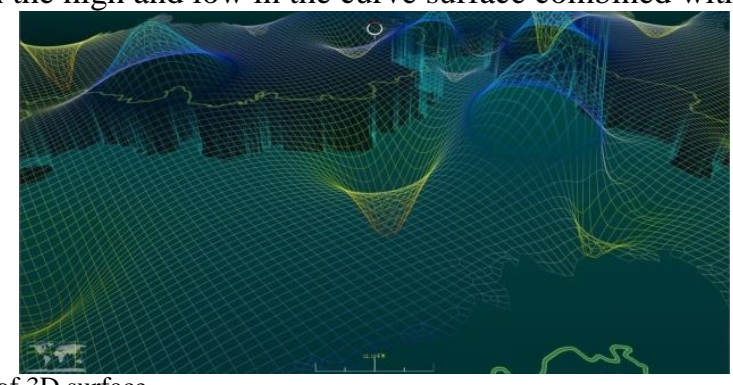

\section{Summary}

The construction goal of failure outage risk assessment platform is unity in platform and functional, flexibility, independent of the application, as well as for the dual interactive ability by visualization. In order to realize the interactive visualization of unified platform and interface between systems, the platform developed a unified service interface based on Web service technology, including system configuration, visual scene, visual window, object and attribute data, visual style, visual instruction, visualization layer and so on.

\section{Acknowledgements}

This work financially supported by State Grid Corporation of China on Research of Smart distribution grid overall view risk management and self-cure technologies of Big Data-oriented which project number is PD71-15-043.

\section{References}

1. He, Jiguang and Hussain, End-to-end outage probability analysis for multisource multi-relay systems, 2016 IEEE International Conference on Communications, ICC 2016.

2. Li Baowei and Ni Chuankun, Analysis Scheme for Relay Protection Fault Visualization in New Generation Smart Substation, Automation of Electric Power System, vol. 38(5). Mar.10,2014, pp.73-77.

3. Wu C L, Hen Z W, Weng Z P, Liu J Q. Property, classification and key technologies of three-dimensional geological data visualization, Geological Bulletin of China, 2011, 30(5):642-649.

4. Zhang Jianmin, Chen Hao, Chen Jian. Smart Grid Situation Awareness Diagram Modeling and Conceptual Design of Situation Awareness 
Visualization, Automation of Electric Power System, vol. 38(9). May.10,2014, pp.168-176.

5. Zhao Lin, Wang Lili, Liu Yan, Research and Analysis on Visualization Technology for Power Grid Real-Time Monitoring, Power System Technology, vol 38(2).Feb.2014, pp.538-543. 